\section{Clozapine-treated NMS}

SIR: Having recently experienced difficulties treating a floridly disturbed young schizophrenic man who had previously had two episodes of neuroleptic malignant syndrome (NMS), we felt it may be helpful to others in this predicament to report our experience with clozapine.

Case report. The patient was a 35-year-old man with an 18 year history of schizophrenia, which for some years had been resistant to a broad range and dosage of established antipsychotics, therapeutic only in that he was a little more in touch with reality and his behaviour rather less disturbed. He had needed several periods of treatment in the secure unit, for unprovoked assaults in his District General Hospital ward. He was receiving treatment with zuclopenthixol (1000 mg weekly), chlorpromazine (200 mg q.d.s.), and procyclidine (5 $\mathrm{mg}$ t.d.s.) when he developed NMS characterised by pyrexia, rigidity, flushing, sweating and agitation with some apparent confusion. Creatine phosphokinase (CPK) levels were significantly elevated (up to 1467 IU/L). His antipsychotic medication was stopped and the NMS abated. He remained settled for a number of weeks but his psychosis returned and required treatment. It was felt that chlorpromazine was worth trying cautiously, but he developed a recurrence of NMS, and all antipsychotics were stopped. Following recovery from NMS, his mental condition deteriorated rapidly with florid delusions, severe thought disorder, and grossly disordered behaviour and aggression, necessitating transfer to the secure unit and treatment in seclusion for an extended period. General sedation with phenobarbitone and benzodiazepines was unsuccessful. We were faced with a difficult dilemma, in that he needed urgent antipsychotic treatment but stood the risk of recurrence of NMS. In view of the recurrence with chlorpromazine, it was felt that most of the well-established drugs were not acceptable (Susman et al, 1988), and in view of its different chemical structure and pharmacological profile (Coward et al, 1989), a decision was made to commence the patient on clozapine, with the usual precautions, plus observations with a view to detecting NMS. Over a period of seven weeks the patient improved markedly in behaviour, thought and contact with reality, and regained some of his emotional warmth. He is as well as he has been for several years, has shown no signs of a recurrence of NMS, and is well enough to return to the open ward.

Apart from being consistent with reports that clozapine is effective in resistant cases who have not responded to other established medication (e.g. Kane et al, 1988) this case suggests that clozapine is a drug to consider when a patient has suffered NMS.

M. F. BURRELL C. FEWSTER

Whittingham Hospital

Whittingham, Preston

Lancashire PR3 2JH
Withington Hospital

West Didsbury

Manchester

Victoria Hospital

Blackpool, Lancashire

\section{References}

COWARD, D. M., Imperato, A., Urwyler, S., et al (1989) Biochemical and behavioural properties of clozapine. Psychopharmacology, 99, S6-S12.

KANE, J., Honigfeld, G., Singer, J., et al (1988) Clozapine for the treatment of resistant schizophrenic. Archives of General Psychiatry, 45, 789-796.

Susman, V. L. \& AdDonizio, G. (1988) Recurrence of neuroleptic malignant syndrome. Journal of Nervous and Mental Disease. 176, 234-241.

\section{All-night television can damage your health}

SIR: Patients with chronic schizophrenia often reverse the more usual night/day pattern of sleep and activity. This enhances their social withdrawal and hinders attempts to engage them in rehabilitation programmes. The provision of all-night television on the independent channels since August 1987 reinforces this behaviour and can have deleterious effects on the mental states of patients if, as a result, they fail to comply with, or attend for, medication. The following case report illustrates how all-night television watching led to a self-limiting symptomatic relapse.

Case report. A 31-year-old Afro-Caribbean male who lived with his parents and sister and who had a 10-year history of paranoid schizophrenia was symptom-free on a weekly depot and twice-daily oral anti-psychotic medication. He attended a day hospital where he received his medication and attended workshop facilities. His generally excellent attendance at the day hospital declined and he regularly missed the mornings, arriving between 1.00 p.m. and 3.00 p.m., complaining of feeling tired. Under close questioning he admitted that he had started watching all-night television until $5.00 \mathrm{a} . \mathrm{m}$. He missed doses of his prescribed oral medication and for some weeks he also missed his depot and started to complain of unpleasant auditory hallucinations. When confronted with his poor attendance and compliance and advised to go to bed earlier so that he could attend a full day at the day hospital, he refused, saying that he enjoyed the selection of programmes available overnight and was not prepared to miss them. After six weeks of poor attendance and missing doses of medication he began to experience auditory hallucinations emanating from his television set, and to feel that people on television were interfering with his thoughts. These experiences made television watching intolerable and, consequently, he began to retire to his bed earlier. His attendance at the day hospital improved to that his prescribed medication could once again be given under supervision, and his symptoms disappeared. 